\title{
Characterization of Metering, Merging and Spacing Requirements for Future Trajectory-Based Operations
}

\author{
Sally Johnson ${ }^{1}$ \\ Adaptive Aerospace Group, Inc., Hampton, VA 23666
}

\begin{abstract}
Trajectory-Based Operations (TBO) is one of the essential paradigm shifts in the NextGen transformation of the National Airspace System. Under TBO, aircraft are managed by 4dimensional trajectories, and airborne and ground-based metering, merging, and spacing operations are key to managing those trajectories. This paper presents the results of a study of potential metering, merging, and spacing operations within a future TBO environment. A number of operational scenarios for tactical and strategic uses of metering, merging, and spacing are described, and interdependencies between concurrent tactical and strategic operations are identified.
\end{abstract}

\section{Introduction}

Trajectory-Based Operations (TBO) is one of the essential concepts in the NextGen transformation of the National Airspace System (NAS) ${ }^{1,2}$ and in the European Union's Single European Sky Air Traffic Management Research (SESAR) effort ${ }^{3}$. TBO utilizes 4-Dimensional Trajectories (4DTs) that span all phases of flight from pushback to arrival at the gate as the basis for planning and executing flight operations. While the final concept of operations for TBO is still being developed, NASA has been supporting the FAA in development and implementation of TBO through initial concept exploration studies ${ }^{4}$ and research projects culminating in a series of technology demonstrations.

In TBO, trajectories are negotiated between the operator and the Air Navigation Service Provider (ANSP), both preflight and during the flight as conditions change, to satisfy the operators' business objectives and preferences while meeting ANSP constraints. User preferences are accommodated to the greatest extent possible, and trajectories are constrained only to the extent required to maximize capacity and accommodate demand or other national concerns, such as safety or security. In high-density or high-complexity airspace, the ANSP may need to limit the aircraft to a given published airway and assign constraints at specific points, while in low- to medium-density airspace, a windoptimal route defined by a series of arbitrary points in space identified by latitude and longitude might be negotiated. The use of precise 4DTs dramatically reduces trajectory uncertainty and enables airspace to be used more effectively to safely accommodate high levels of demand, reduce environmental impacts, and maximize the use of capacitylimited airspace and airport resources.

Airborne- and ground-based metering, merging and spacing (MMS) operations play a major role in TBO. If the destination airport or en route airspace is capacity-constrained, gate-to-gate flow may be managed by subjecting flights to time-based constraints, handled via an airborne or ground-based MMS operation. MMS operations can also be used tactically to ensure smooth flows and to increase capacity and flexibility without undue increases in controller workload. As weather and traffic conditions evolve during the flight, time-based constraints are modified to maximize use of constrained airspace resources and new trajectories are negotiated. Thus, although flights are managed via 4DTs from preflight planning through all phases of flight, TBO does not place unnecessary constraints on the flight, but rather provides for flexibility to deal effectively with uncertainties in the system, such as weather. The goal is for operators and service providers to collaboratively negotiate aircraft trajectories, providing agile, optimized, aircraftspecific routing to meet gate-to-gate flow-management constraints, and increasing capacity by smoothly and effectively combining flight-deck-based and ground-based metering, merging, and spacing in a mixed-equipage environment.

This paper focuses on an initial set of example applications of flight-deck-based and ground-based MMS in a mixed-equipage environment that could be implemented in a mid-term TBO environment. This study, which leverages previous research findings, is an initial step toward defining a candidate set of functional requirements for onboard and ground-based tools and procedures to enable a suite of MMS operations appropriate for different situations and operational goals across the en route, departure, and arrival phases of flight. First, the characteristics of today's typical flight are examined, with an eye towards optimization criteria. Then, a set of likely tactical and strategic MMS operations are identified for aircraft that are on established airways or utilizing an optimized, aircraft-

\footnotetext{
${ }^{1}$ Aerospace Engineer, member.
} 
specific routing. Within this paper, tactical refers to operations that are in effect within one sector or perhaps a few adjacent sectors, while strategic refers to MMS operations that are in effect across multiple sectors and may even encompass several hours of flight time. The characteristics of the MMS operations are analyzed, and interdependencies between tactical and strategic operations are identified, to begin the process of defining requirements for an integrated suite of MMS algorithms and procedures.

\section{Characteristics of Flight in Today's NAS}

This section describes the operational characteristics and optimization criteria for a typical civil air transport aircraft on a U.S. transcontinental domestic flight to lay the basis for later sections examining how the characteristics of this flight profile impact the feasibility and usefulness of tactical and strategic operational improvements under today's operations and during transition to TBO.

\section{A. Flight Vertical Profile}

A typical vertical profile for a civil air transport on a U.S. transcontinental flight is shown in Figure 1. Once the aircraft lifts off the runway, it accelerates to the initial climb speed. The initial climb may be governed by airportspecific noise abatement procedures in addition to the $250 \mathrm{kt}$ Calibrated Air Speed (CAS) speed limit below 10,000 $\mathrm{ft}$. Once the aircraft climbs above 10,000 ft., it accelerates to the en route climb speed for the aircraft. At each point during the flight, there is an optimum cruising altitude and a maximum altitude limit for the aircraft at the current weight, based on the coefficient of lift, $\mathrm{C}_{\mathrm{L}}$, at each altitude, which is dependent on a number of atmospheric parameters. These altitudes increase as the aircraft weight decreases due to fuel consumption. Most airliners are equipped with a Flight Management Computer that continually calculates the optimum and maximum flight levels based on aircraft weight, which is adjusted as fuel levels decrease. Decades ago when air travel was new and traffic was light, civil air transports typically flew cruise-climb vertical profiles, where the aircraft is continually gradually climbing to higher altitudes for optimal cruise efficiency, but daytime clearance for cruise climb is rare in today's NAS. Today, aircraft are constrained to level flight at specific altitudes and must request clearance to climb to the next available altitude, called a step climb, each time sufficient fuel is consumed. While a flight profile using step climbs is not as fuel efficient as cruise climb, the inefficiency is minimized when step climbs can be performed at optimal times for the flight. The first time the aircraft levels off is considered the Top of Climb. Our transcontinental U.S. flight is in en route cruise (from Top of Climb to Top of Descent) approximately five hours and makes two step climbs, slightly more than one hour apart. The pilot would be unlikely to request a step climb during the last hour of en route cruise, since the amount of time the aircraft would be operating at the higher altitude would not produce enough fuel savings to offset the fuel burned to reach the higher altitude. Shorter flights make zero or one step climbs, and some very short hops might not complete climbing before it is time to begin their descent. With today's use of Reduced Vertical Separation Minimum (RVSM) operations, most step climbs will be 2,000 ft altitude change.

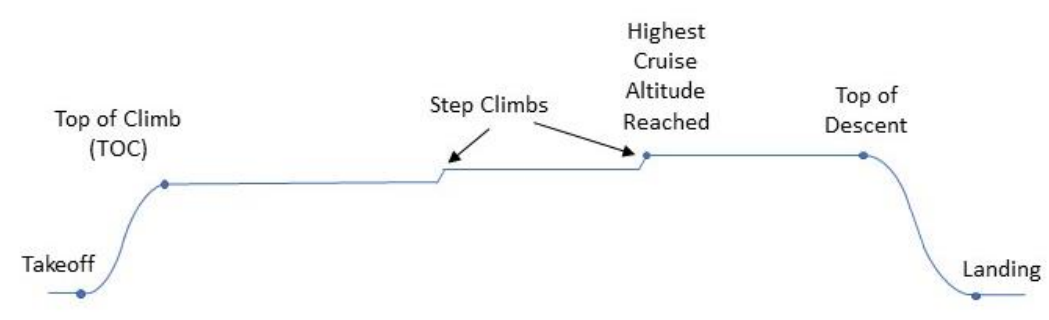

Figure 1. Vertical Profile for Civil Air Transport Flight

Altitude changes may also be made during the en route portion of a flight to avoid hazardous weather or atmospheric turbulence or possibly even to avoid traffic aircraft. For simplicity, the altitude-change operations in this document are generally described in terms of climbing, but the same MMS operations could be performed during descents.

\section{B. Cruise Speed}

During takeoff and climb, the aircraft flies at a continually increasing CAS, measured in knots, until it reaches a set Mach speed, where the Flight Management Computer transitions to measuring speed in Mach, which is the ratio of the aircraft's speed divided by the speed of sound. The speed of sound is affected by atmospheric conditions such as temperature, atmospheric density, humidity, and the presence of moisture, with approximate speeds ranging from 661 knots at sea level to 602 knots at 25,000 ft to 573 knots at an altitude of 40,000 ft, and little change above 40,000 
ft. Thus, as the aircraft changes altitude in en route cruise and maintains a constant Mach speed, this translates to slight differences in groundspeed below an altitude of approximately 40,000 ft. There can also be significant differences in wind speed and direction at different altitudes, which also affect ground speed.

\section{Acceptable Variations in Vertical Profile and Speed}

For an aircraft in en route cruise from Top of Climb to Top of Descent, it is assumed that a reasonably small speed variation would be acceptable. According to the specifications for a Boeing 757 aircraft, Mach .86 is the maximum cruise speed, and Mach .8 is the most economical cruise speed, or "Econ speed." Thus, if the flight takes off on time and no delays are expected en route, then the pilot will want to fly at Mach .8, but if the takeoff was significantly delayed, the pilot might want to fly close to Mach .86, which is a 7.5\% increase in speed. For this study, it is assumed that the pilot/airline would also be willing to accept the same percentage decrease in speed if needed by air traffic control for an en route cruise leg of the flight, say 7.5\% slower than Econ speed, or Mach .74 for the Boeing 757 aircraft. Just prior to descent, a more significant reduction in speed might be acceptable, but early in the en route phase a controller request for prolonged operation at a substantially slower speed might result in the airline requesting to deviate to a different route that could be flown with a more expeditious speed.

Similarly, it is assumed that in some situations an aircraft will be stuck at a less-than-optimal altitude for a significant period of time because higher altitudes are blocked by traffic aircraft. It is assumed that an en route delay in climbing of 20 to 30 minutes might be accepted, but if it is clear that dense traffic on the current route will impede climbing for a longer period of time, the airline might request a deviation to a less dense route.

\section{Flight Lateral Profile}

Today's transcontinental flights are typically restricted to a limited set of predefined airways that the Air Traffic Control System Command Center (ATCSCC) has preselected for the departure/arrival city pair for that day. The airline dispatcher will request a route from among the options that takes advantage of predicted winds aloft, such as following the jet stream on an eastbound flight or encountering the least strong headwinds on a westbound flight. Once the aircraft takes off, if the traffic density is low enough, the pilot may be able to occasionally request to fly direct to a future waypoint to cut off a few corners from the predefined airways, but large routing changes are not typical unless needed to deviate around unexpected weather or areas of high traffic density, which is usually coordinated by the dispatcher.

If the aircraft is arriving at a major airport or metroplex, today's route likely includes a detour several hundred miles out of the way to enter a dense queue of traffic heading towards the destination. The controller managing the aircraft entering the queue in today's NAS must handle multiple aircraft arriving at once from different directions at diverse speeds that must all be slowed and routed using short or long path stretches to deliver them into the queue with proper spacing between them. A short path stretch might be a 10-mile fixed radial deviation, where the aircraft is turned to a heading to the right or left of the airway and then after traveling for 10 miles is directed back to join the airway, having absorbed several minutes of delay. A longer path stretch would be a deviation of a longer distance defined by a set of headings and/or waypoints. Flow management procedures, such as Miles-In-Trail, may be employed to limit the number of aircraft arriving in a sector within a given 15-minute period. However, the timing of sector arrivals is not necessarily evenly spread, so many of those aircraft may arrive at one time from different airways and may all be headed to the same destination. There is almost no attempt in today's NAS for a sector controller to anticipate or try to pre-empt complex conflicts that will arise in a downstream sector. In any high-density airspace, it is not unusual for a controller to use small path stretches in addition to speed clearances to maintain proper spacing between aircraft or to open up a gap for another aircraft to merge onto the airway.

\section{E. Weather Disruptions}

Weather can cause huge disruptions in the NAS for two reasons: 1) there is significant uncertainty associated with weather prediction, and 2) weather can stretch across hundreds of miles, causing NAS-wide impacts. While current efforts to improve weather information-gathering and prediction methods and to share weather information with all stakeholders through System-Wide Information Management (SWIM) will improve the flight planning process, weather predictions will remain uncertain in terms of when, where, and how severe the weather conditions will evolve. Widespread severe weather obviously can be extremely disruptive to NAS operations, but even localized weather issues pose challenges for capacity and efficiency. The presence of low ceilings significantly impacts arrival and departure rates at airports, and localized severe convective weather can block arriving and departing or en route streams of traffic.

The ATCSCC oversees the management of the timing and routing of flights before they depart, with a look-ahead horizon of 2-15 hours, focusing on ensuring that demand will not exceed capacity in any sectors or terminal areas 
across the NAS. When traffic demand for an area of en route airspace is projected to be above capacity due to established airways being blocked by convective weather, a Flow-Constrained Area (FCA) is established and flow constraint procedures are employed to limit the number of aircraft entering the area. Flow constraint procedures include ground delays and ground stops for aircraft that have not yet taken off plus Miles-in-Trail restrictions that slow the rate of aircraft entering the capacity-constrained region of airspace by applying large spacing distances between aircraft along an airway approaching the region. Flights that cannot be accommodated within the FCA can also be rerouted to avoid the area, and airlines may choose to cancel affected flights.

Geographically dispersed Traffic Management Units (TMUs) manage local en route and terminal area traffic flow for flights that are already in the air. Disruptions, such as hazardous weather or reduced throughput due to Instrument Meteorological Conditions (IMC), are handled manually by moving groups of aircraft, with limited negotiation with airlines to prioritize flights.

The effects of weather disruptions on airline operations are profound. Delayed flights mean missed connections and crew duty-hour constraints limit recovery plans. Canceled flights mean stranded passengers and airplanes not positioned appropriately for the schedule, so recovery can ripple into the next day's operations. Typically, when a flight has been held on the ground because the destination airport is capacity-constrained, time is now much more important to the airline than fuel efficiency, and once the flight takes off, the pilot will fly as fast as allowed to try to recover the schedule. Unless blocked by other aircraft ahead, the pilot has discretion to increase or reduce speed up to five knots or five percent, whichever is greater, relative to the speed assigned in a clearance. However, once the aircraft enters the dense queue of aircraft headed towards the capacity-constrained destination, first come first served is the dominant rule, and the aircraft is likely to incur even more delay in the form of reduced speeds, path stretches, and even airborne holding 5 .

\section{Metering, Merging, and Spacing Algorithms and Enabling Technologies}

The MMS operations considered in this study include both flight-deck-based procedures conducted by a pilot, and analogous ground-based procedures conducted by a controller. Metering is the use of time-based scheduling at a specific control point to deliver a smoother and more regulated flow of traffic. Metering can be performed over short time horizons, but is more useful over longer, strategic time horizons. Merging and spacing is an operational flow concept based on relative time between aircraft rather than managing to absolute time. Since merging and spacing is a pairwise, aircraft-specific operation, it is more effectively used over short time horizons. Merging and spacing can impose necessary constraints on a flight while allowing more flexibility in traffic flows than time-based metering with tight time boundaries.

\section{A. Metering Operations}

Metering today is typically accomplished by a controller using speed guidance calculated by ground-based decision-support tools, which is communicated via voice to the pilot as one or more speed clearances. In a future TBO environment, some aircraft will still be receiving speed clearances from the controller via voice or datalink, while other aircraft with advanced equipage will be cleared to meet the time constraint via a Time Of Arrival Control (TOAC) operation. Flight-deck-based TOAC could be performed using Required Time of Arrival (RTA) functionality within the aircraft's Flight Management System (FMS) or potentially using TOAC guidance implemented on an Electronic Flight Bag (EFB), which would provide speed guidance to the pilot. In the TOAC operation, an aircraft is assigned a time window to cross a specified waypoint. The FMS RTA capability calculates the speed required to achieve the RTA within the defined window, and notifies the pilot when unable to achieve the TOAC timing.

There have been a number of flight trials to explore the use of RTA functionality for trajectory management and TOAC. Studies in 2001-2008 showed that existing RTA technology can reliably predict and control to an RTA in en route and terminal airspace ${ }^{6,7,8,9}$. The FAA conducted two sets of RTA flight trials in 2010 and $2011^{10}$, demonstrating that aircraft can meet desired RTA time performance to deliver aircraft to the Scheduled Time of Arrival (STA) assigned by ground-based schedulers, but there were a number of operational issues uncovered in implementing TOAC in the NAS. Only $71 \%$ of the flights that were assigned an RTA fully executed the RTA within time tolerance to the meter fix, with $16 \%$ canceled by the controller. Operational issues included controller and pilot concerns with RTA speed profiles, pilot workload in managing speeds and auto-throttles, controller workload in managing spacing in heavy traffic, and the "fragility of RTA under current operating conditions." In Europe, flight demonstrations were performed in 2013 to demonstrate the Initial 4D trajectory management concept developed under SESAR's TimeBased Operations concept, demonstrating the use of uplinked RTAs for TOAC and downlinked messages for trajectory synchronization between stakeholders ${ }^{11}$. While all of these studies point to the likely eventual operational and technical feasibility of TOAC operations for managing flow, a number of operational issues were commonly 
reported that must be resolved, including controllers feeling the need to maneuver other aircraft out of the way to clear the way for the test aircraft. NASA's research in implementation of TOAC will build on the lessons learned from these previous studies.

Previous research has shown that, since the aircraft's FMS is the most accurate predictor of the trajectory, an effective procedure for assigning the STA is for the pilot to use the FMS to generate a window for its expected arrival at the control point and communicate that to the controller so that a feasible RTA can be assigned within that window. TOAC could be applied in both tactical (less than 30 minutes out) and strategic (more than 30 minutes out). Today's FMSs can only manage to one RTA at a time; however, multiple TOAC points during a flight that are separated by substantial amounts of time could perhaps be handled by the FMS one at a time, as long as the timing is consistent so that managing to an earlier RTA does not jeopardize meeting subsequent ones.

The use of RTA capability to conduct TOAC operations is subject to a number of limitations. The RTA algorithm does not consider the existence of traffic aircraft, but rather assumes that the aircraft can freely speed up or slow down on the route. Different FMSs use different algorithms to achieve the RTA, with inconsistent timing characteristics; for example, aircraft on the same route performing speed management to deconflicted RTAs at a future point will likely not stay evenly spaced on the route. A civil transport aircraft has a limited speed range and limited range of accelerations, so relatively long periods of operational time are needed to catch up or slow down to meet an RTA.

\section{B. Merging and Spacing Operations}

Ground-based merging and spacing operations require ground-based decision-support automation to generate speed clearances that controllers send to aircraft via voice or future datalink communications. Flight-deck-based merging and spacing is an operation in which a pilot uses onboard Automatic Dependent Surveillance-Broadcast (ADS-B) In information and algorithms and displays to perform the operation, and may also receive uplinked route and timing information for one or more other aircraft to support onboard calculations. Much has been learned through decades of research ${ }^{12,13,14}$ about how to effectively implement merging and spacing, focusing primarily on arrival and oceanic operations. The concept can be extended to manage en route flows, building on what has been learned in other domains.

Terminal area merging and spacing research and development is quite mature ${ }^{15,16}$, with scheduling tools and ground-based decision support automation to provide controllers with speed guidance beginning operational implementation and with standards developed for airborne algorithms and procedures. Measurable improvements in management of terminal-area traffic flows have been demonstrated by the deployment of Time-Based Flow Management (TBFM) ${ }^{17}$, based on NASA's Traffic Management Advisor (TMA) ${ }^{18}$, which conditions the flow of arrivals into a busy airport. TBFM predicts the trajectory of each aircraft along its projected descent path, detects spacing conflicts, and generates information to support the controller in handling any spacing issues. The scheduled times of arrival of each aircraft are allowed to change until they cross the freeze horizon (an arc prior to the top of descent), at which time the aircraft's sequence and scheduled times of arrival are frozen. NASA has been working with the FAA to investigate the integration of merging and spacing operations with TBFM to support both groundbased and flight-deck-based merging and spacing of aircraft on arrival ${ }^{19,20}$. These near-term improvements are only precursors to TBO, and current operations are far below the traffic throughput and efficiency benefits to be gained from the transition to gate-to-gate TBO. Site adaptation issues and performance limitations of these improvements ${ }^{21}$ have provided valuable evidence of the importance of a system-wide approach to flow management with integration of ground-based tools across sectors and the need for system-wide optimization.

In terminal area merging and spacing, aircraft arriving at an airport from multiple routes are synchronized with sufficient gaps in spacing to allow each aircraft to smoothly fit into the converging flow while maintaining the minimum spacing required between all aircraft. To maximize runway utilization, the terminal-area spacing algorithm is designed to deliver the aircraft close to the minimum spacing at the final spacing control point. In this document, algorithms that are designed to deliver aircraft at a specific spacing will be referred to as Achieve By algorithms, which must achieve the designated spacing by a specified point in the trajectory and Maintain algorithms, which maintain the designated spacing within tolerance once the designated spacing interval is achieved.

In en route airspace, there are some situations where it is desirable to close up the inter-aircraft spacing along a route to a minimum time at a designated control point, such as the entry point of a region of an FCA. There are also situations where it is desirable to open up the spacing between a pair of aircraft on an airway, such as to allow another aircraft to merge onto the airway. In both of these situations, a combination of an Achieve By operation and a Maintain operation would be used.

For the flight-deck-based Achieve By operation, the aircraft is assigned a time to achieve behind a target aircraft at or before arrival at a specified waypoint. The flight-deck-based Achieve By algorithm compares the ownship's predicted time to the waypoint to the target aircraft's predicted time to the waypoint and calculates the speed required 
for the ownship to achieve the designated timing behind the target aircraft at the specified waypoint. The algorithms are typically implemented with a limited speed range, so relatively long periods of time are needed to catch up or slow down for spacing. After reaching the specified waypoint, the onboard automation would typically switch to a Maintain spacing algorithm. In this operation, the ownship aircraft is already at the desired timing behind the target aircraft and maintains that relative timing until arrival at a future waypoint. The Maintain spacing algorithm compares the measured spacing to the target aircraft and calculates the speed required to stay at the assigned spacing. For the Maintain spacing operation, the two aircraft must be on the same path or on parallel to slightly converging paths.

In the ground-based Achieve and Maintain operation, the controller identifies a leader-follower pair of aircraft and uses decision-support automation to periodically calculate the speed required for the follower aircraft to arrive at a specified waypoint with the desired timing behind the leader aircraft. Speed modifications are periodically transmitted to the pilot of the follower aircraft via clearances. The leader and follower aircraft do not have to be on the same 3D route, but the ground-based automation must have information on both aircraft's routes to calculate the timing. Since the ground-based operation is handled through clearances, speed changes are typically less frequent and in larger speed increments than in flight-deck-based operations.

Previous oceanic spacing research and development, including the In-Trail Procedure $22,23,24,25$ and Pairwise Trajectory Management (PTM) ${ }^{26,27,28}$, has extensively studied spacing between aircraft during oceanic level flight and altitude-change operations, including both on and off oceanic track systems. Many of the previous studies conducted can be leveraged in the development of en route merging and spacing, including development and evaluation of procedures, clearance messaging, and pilot interfaces, as well as safety analyses and analyses of the impacts of ADS$\mathrm{B}$ signal range. While oceanic operations and surveillance differ significantly from domestic en route, much can be learned from the previous oceanic airborne spacing research, such as calculating and displaying to the pilot a range of speeds that would allow an aircraft to maintain desired speeds and make desired altitude changes while maintaining at least the required minimum spacing from designated aircraft.

Spacing algorithms designed to allow maximum flexibility while ensuring minimum spacing requirements are not violated are referred to in this document as No-Closer-Than algorithms. In much of en route airspace, traffic density is low enough to allow aircraft some flexibility to fly their desired speeds as long as spacing conflicts do not arise. When closing up the spacing interval is not a goal, an aircraft that is satisfactorily spaced behind a target leader aircraft and in front of a target follower aircraft could be cleared for a flight-deck-based No-Closer-Than operation to maintain safe spacing intervals until the operation is terminated. The aircraft do not have to be on the same route, so this could also be used for intersecting traffic for a limited range of angles. The aircraft would not have to be co-altitude, so this operation can be used during climbs and descents. The algorithm receives ADS-B information from the two target aircraft and calculates a range of ownship speeds that will satisfy the spacing criteria, and the range is displayed to the pilot.

The ground-based spacing operation utilizes decision-support automation that provides speed guidance to the controller and provides conformance monitoring to maintain safe spacing intervals between pairs of aircraft without a designated target spacing interval to achieve. Like the flight-deck-based operation, ground-based spacing could also be used during climbs and descents and for intersecting traffic for a limited range of angles.

\section{Implementation Criteria}

As new ground-based and airborne MMS functionality is implemented, it is important that these new functions be seamlessly integrated into existing systems. In order for a controller to effectively use these operations in mixedequipage airspace to increase traffic throughput and improve predictability while better meeting user business objectives, the controller will require new decision-support functionality both to implement ground-based operations and to facilitate clearing and monitoring of flight-deck-based operations. Conformance monitoring also plays an important role in effective use of MMS operations, including onboard alerting when an aircraft is trending toward or predicted to imminently be out of conformance with a timing requirement as well as conformance-monitoring functionality for ground-based MMS operations embedded in decision-support automation. An integrated suite of onboard algorithms suited to different types of MMS operations will likely be required, with a single interface and similar procedures between operations to avoid confusion. 


\section{MMS Operations in a TBO Environment}

Under TBO, airborne- and ground-based MMS operations play a major role in managing aircraft by precise 4DTs to reduce trajectory uncertainty and inefficiency, enable airspace to be used more effectively to safely accommodate high levels of demand, and to maximize the use of capacity-limited airspace and airport resources. While in-depth studies are needed to determine the most effective uses of MMS in the mid-and far-term TBO environment, they have tremendous potential to address many of the problems encountered in today's NAS operations. If a destination airport or region of en route airspace is capacity-constrained, gate-to-gate flow management may be handled by subjecting individual flights to time-based constraints, resulting in improved conditioning of the flows into high-density areas with more fuel-efficient trajectories and opportunities for resequencing aircraft for fleet management priorities through Collaborative Air Traffic Management (CATM). Development of new procedures and equitable enforcement policies for time-based management could eliminate the current practices of aircraft being held on the ground and then flying as fast as possible to make up time. Improved flow management schedules and procedures will balance the need to keep pressure on capacity-constrained resources so they are fully utilized while minimizing the need for airborne holding and inefficient routing.

MMS operations can also be used tactically to ensure smooth flows within a sector and perhaps to increase capacity and flexibility without undue increases in controller workload. By managing individual aircraft through time-based operations, whether conducted by the flight deck or through increased ground-based functionality, MMS operations may have the potential to safely increase the density of a controller's sector through workload mitigation, enabling the creation of new additional airways and perhaps enable more use of user-defined routes off of predefined airways. The FAA is developing a concept for Dynamic Required Navigation Performance (D-RNP), where aircraft are rerouted by the controller to dynamically defined routes by uplinking a 4D trajectory.

A key research question in the transition to TBO is the role of path stretches in future NAS operations. The use of tactical and strategic MMS operations to act preemptively to provide more predictable, smooth flows of aircraft may substantially reduce the need for path stretching in many situations. However, if path stretches do still occur in the future NAS, which is likely, then the MMS operations must be designed to work properly in combination with path stretches. While pairwise MMS operations along well-defined paths should be straightforward to implement, some situations that would be encountered frequently in today's NAS would be quite challenging, including spacing behind an aircraft that 1) is performing a path stretch, 2) has be vectored to avoid a traffic conflict, or 3) is maneuvering off-course to avoid hazardous weather.

\section{A. Arrival/Departure MMS}

MMS can be used in arrival/departure operations to address conditioning the flow of aircraft approaching the extended terminal area to match traffic density to airport arrival rates, spacing of aircraft on approach and departure corridors, and metering of departing traffic into high-density en route streams. Time-based scheduling and management of arrivals is already being operationally implemented for a few major airports. Early results are promising, but managing complex merging flows of long and short flights in today's environment requires the use of complex scheduling techniques with multiple freeze horizons to condition the flow at multiple points on arrival. One of the goals of TBO research is to develop more effective strategic MMS operations to make the flows of aircraft more smooth and predictable. With better flow management and improved merging operations, the long, dense queues flowing into major airports should be substantially shortened in far-term TBO or even sooner. Key challenges for arrival/departure operations include designing MMS operations to support Continuous Descent Approaches and Optimized Profile Climbs for aircraft in streams of mixed-equipage traffic and continuing MMS operations in the presence of high traffic demand and disruptions, such as missed approaches and minor deviations for weather within a predefined corridor.

\section{B. Tactical En Route MMS}

MMS operations can also be used tactically to ensure smooth flows and to increase capacity and flexibility without undue increases in en route controller workload. Controllers currently separate and manage air traffic manually with limited decision-support automation, using radar screens to display streams of traffic on predefined airways and making cognitive operational judgments to ensure safe and efficient traffic flows. Controllers become experts at efficiently handling the specific flow patterns within their sectors, quickly examining streams of traffic to ensure safe spacing and managing the interleaving of traffic at intersections and merge points. TBO may enable greater use of aircraft-specific trajectories off of established airways by minimizing the controller workload for monitoring these aircraft through pairwise airborne MMS operations to off-airways aircraft for crossing streams of traffic or merging onto airways, combined with appropriate ground-based decision-support and conformance monitoring automation. 
MMS operations might also be used for multiple aircraft picking their way through a gap in the weather, increasing the throughput of aircraft through the gap and alleviating controller workload for managing the aircraft that are shooting the gap. Alternatively, ground-based decision support tools may be developed to help the controller manage aircraft-specific trajectories at the same workload as needed for today's published airways without using flight-deckbased MMS operations.

\section{Strategic En Route MMS}

Under TBO, a combination of capacity enhancement and flow management procedures will be employed. When traffic demand for a region of en route airspace is projected to be above capacity due to established airways being blocked by convective weather, the first step is to try to enhance the capacity of the region by activating additional predefined airways or by dynamic creation of new airways, using tactical MMS operations as applicable to manage controller workload in each sector. If capacity enhancement procedures alone are insufficient, then a Flow Evaluation Area will be established to notify operators of the capacity constraint. If flight cancellations and reroutes are still insufficient to manage the demand/capacity imbalance, then a Flow-Constrained Area (FCA) will be established. Instead of ground holds and Miles-in-Trail restrictions, strategic MMS operations will be employed to smoothly manage the flow of aircraft into the FCA while supporting user priorities through CATM.

\section{Combinations of Tactical and Strategic MMS}

Gate-to-gate flow management is a complex mix of local and global optimization. The flow within a local area of high-density airspace must be optimized, and the flow into the high-density area needs to be managed to ensure safe and efficient flights. Thus, an individual flight may be simultaneously subject to both a tactical MMS operation to aid the controller in managing the local flow, and a strategic MMS operation such as a TOAC near the destination airport. Section VI discusses the interdependencies between tactical and strategic MMS operations.

\section{Tactical Merging and Spacing Operations}

While much research remains to be conducted, this section examines some examples of tactical MMS operations that could be performed by aircraft to both reduce controller workload and to enable operations that might not be approved by busy controllers in today's environment. All of these operations require that the flight crew must be trained and current, and the pilot may reject the clearance as "unable" if the timing of the operation is deemed to be risky or infeasible. The ownship must have ADS-B In and onboard algorithms for calculating and displaying to the pilot the allowable range of speeds for conducting the operation. The designated Leader and Follower aircraft must be equipped with ADS-B Out. Whether the operations involving spacing or merging with aircraft that are on the same airway, or on a different airway, the ownship onboard algorithms must have a means of determining the relative time between the ownship and designated Leader and Follower aircraft to key control points, such as airway intersections, as the operation progresses.

\section{A. Single-Altitude, On-Airway MMS Operations}

This section examines MMS operations that could be performed by aircraft on airways to both reduce controller workload and to enable operations that might not be approved by busy controllers in today's environment.

\section{Single-Altitude, On-Airway Airborne Spacing Operation}

A controller managing aircraft on an airway could clear an aircraft to perform airborne spacing, which would reduce workload since he would not have to monitor the spacing for that aircraft. As shown in Figure 2, the aircraft may be cleared for airborne spacing behind a Leader, in front of a Follower, or both.

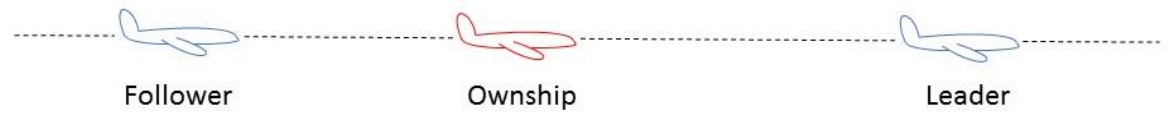

Figure 2. Airborne Spacing on an Airway

If the operation designates both a Leader and a Follower, then the pilot is responsible for keeping the ownship behind the Leader with at least the designated minimal spacing and ahead of the Follower with at least the designated spacing. The two designated spacings may be different timings; for example, the controller may be preserving a gap that will be filled later by another aircraft. The controller may assign one of the designated spacings as a target to achieve by 
a certain time or location, even though that designated spacing is not met when the operation is initiated; for example, the controller may be opening up a gap to later merge another aircraft into the string.

For spacing behind a Leader with no Follower, the pilot could choose from a wide range of speeds. He could fly at a faster speed than the Leader and close up the distance behind the Leader until he reaches the minimum spacing defined for the procedure, at which point he would need to slow to the same speed as the Leader. If his preferred speed is slower than the Leader, then he would gradually fall farther and farther behind the Leader. If the operation designates a Follower but no Leader, then the ownship would be free to fly as fast as desired unless other constraints are put on the flight.

\section{Single-Altitude, On-Airway Airborne Merging and Spacing Operations}

A controller managing aircraft on two converging airways could clear an aircraft to perform airborne merging and spacing, which would reduce workload since he would not have to monitor the merging and spacing for that aircraft. This operation is similar to the single-altitude on-airway spacing operation described above, but the aircraft are spread across two airways that converge or intersect. The aircraft may be cleared for airborne spacing behind a Leader, in front of a Follower, or both, as shown in Figure 3. The Leader and Follower may be on the same airway as the Ownship or on the converging airway. For the aircraft on the converging airway, the spacing is based on relative distance to the merge point rather than physical in-line distance.

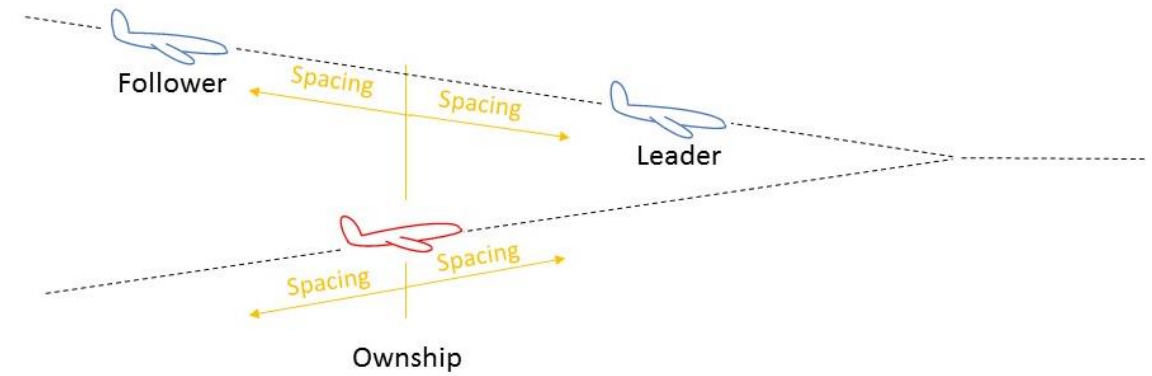

Figure 3. Airborne Merging and Spacing on Two Converging Airways

The operation could be initiated before the ownship has achieved the designated spacing range (for example when the relative distance to the merge point between ownship and Leader is smaller than the designated spacing) as long as the two aircraft are sufficiently laterally separated and the ownship is projected to achieve the correct spacing before lateral separation is lost between the converging airways. The ownship pilot would have to accept the spacing clearance, which affirms his ability to obtain the required spacing (based on guidance from onboard algorithms). Similar to the spacing operation above, the pilot could choose from a range of speeds.

\section{Single-Altitude, On-Airway Intersection Merging and Spacing Operations}

A controller managing aircraft on two intersecting airways could clear an aircraft for airborne merging and spacing for all or part of the intersection merging and spacing. In this operation, the aircraft are spread across two airways that intersect. The spacing is based on relative time to the intersection between the ownship, Leader, and Follower aircraft. The aircraft may be cleared for airborne spacing behind a Leader, in front of a Follower, or both, on the same airway and on the intersecting airway, as shown in Figure 4.

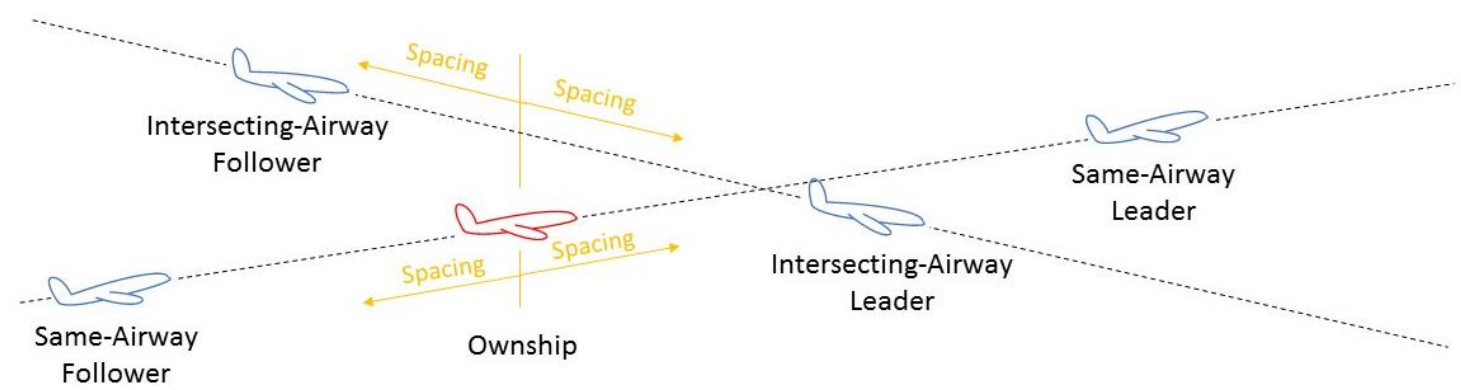

Figure 4. Airborne Merging and Spacing at an Intersection of Two Airways 
The Leader and Follower may be on the same airway as the ownship or on the converging airway. The operation could actually begin before the ownship has achieved the designated spacing range (for example when the relative distance to the merge point between ownship and Leader is smaller than the designated spacing) as long as the two target aircraft are sufficiently laterally separated and the ownship is projected to achieve the correct spacing before lateral separation is lost between the converging airways. As always, the ownship pilot would have to accept the airborne spacing clearance, which affirms his ability to obtain the required spacing (based on guidance from onboard algorithms).

\section{Discussion of Single-Altitude, On-Airway MMS Operations}

Airborne on-airway spacing by a single aircraft would have a minimal impact on controller workload, but airborne spacing by a string of aircraft on an airway segment would reduce the need to monitor that entire segment, having a larger impact on controller workload. Since the Leader and Follower aircraft need only have ADS-B Out, not all aircraft in the string would have to be equipped for MMS, as long as there are no two unequipped aircraft in a row in the string. Airborne merging and spacing by a string of aircraft on two converging or intersecting airway segments would have a more substantial impact on controller workload by reducing the need to monitor each airway segment and the intersection. Using the operation to open up a gap for a merging aircraft prior to a specified waypoint may also prove quite useful to a controller.

Extensive performance and feasibility studies will be required to develop these en route MMS operations. Previous research conducted in support of terminal area merging and spacing demonstrated the feasibility of strings of aircraft providing airborne merging and spacing while slowing on arrival. Studies performed during development of the PTM concept have examined the performance and safety aspects of strings of aircraft performing airborne spacing on merging and intersecting tracks that are more relevant to en route operations, but not with the traffic density or complexity required for en route operations. Merging and spacing operations on approach work well in part because the stream of traffic is compacting naturally as the traffic slows during the descent. The stability of streams of traffic operating en route on high-density airways will need to be studied further - a single pairwise operation might work quite well, but pairwise spacing along a lengthy string of traffic may prove to be more problematic. If spacing is closing up ahead and behind, there may be nowhere to go. Onboard tools can monitor the situation and identify trends before separation is lost, but this still begs the question of how would the controller fix the problem once it is identified within the limits of his workload. If the controller could not solve the problem by sending one aircraft to a different route, he would have to divert multiple aircraft and notify everyone individually.

PTM studies developed algorithms for intersecting tracks, ensuring that separation is maintained between aircraft both laterally as well as longitudinally near an intersection for a limited range of angles between airways and along a track where the track has up to a 45-degree turn angle. This includes analysis where one or both aircraft are offset from the center of an airway using the Strategic Lateral Offset Procedure. A complete set of rules for initiating and maintaining strings of aircraft performing PTM spacing have been developed. PTM also demonstrated the efficacy of a spacing algorithm that would calculate and display to the pilot a range of allowable speeds. The pilot would then choose a speed within the range that satisfies other criteria, such as Econ speed or a faster speed to make up time on a late flight or to meet a downstream RTA. A PTM pilot usability study also revealed that pilots strongly preferred that the clearance include the minimum speed the pilot should expect during the operation when trailing the target or the maximum speed to be expected when in front of the target to aid them in determining the feasibility of the operation.

Much research remains to be performed to evaluate the feasibility of merging and spacing operations on highcapacity airways in en route airspace. Algorithms and procedures would need to be developed to monitor the stability of strings, identifying problematic trends in inter-aircraft pair spacings in time to apply corrective actions before hazardous operational conditions arise.

Implementation and regular use of airborne on-airway merging and spacing operations would likely yield minimal benefits to airlines, such as choice of a slightly more optimal speed on a route. If use of MMS result in more predictability and stability, this may reduce the use of path stretching on high-capacity airways, increasing fuel efficiency and reducing pilot workload. Performance studies, combined with safety analyses, may potentially show that at high equipage levels, airborne merging and spacing along an airway may enable a sector capacity enhancement through reduced spacing on the airway or allowing the opening of other airways in the sector, which would benefit all aircraft operating in that sector. Unequipped aircraft would likely see little to no difference in operations other than minor benefits from any capacity enhancements that are enabled by reduced controller workload. 


\section{B. Altitude-Change, On-Airway MMS Operations}

This section examines MMS operations that could be performed by aircraft on airways during altitude change maneuvers to both reduce controller workload and to enable operations that might not be approved by busy controllers in today's environment.

\section{On-Airway Airborne Spacing with Altitude-Change Operation}

When an MMS-capable aircraft on an airway requests to climb or descend to a new altitude, the altitude-change clearance could include spacing at both the current altitude and the target altitude. The relative spacing is based on the longitudinal distance between the two aircraft, ensuring that a gap is maintained at both the original altitude and the target altitude until the aircraft is established at the target altitude and the airborne spacing operation is terminated by the controller or continued as a single-altitude spacing operation. The aircraft may be cleared for airborne spacing operations relative to up to four traffic aircraft, as shown in Figure 5:

- behind a Leader aircraft at the current altitude,

- behind a Leader aircraft at the target altitude,

- ahead of a Follower aircraft at the current altitude, and

- ahead of a Follower aircraft at the target altitude.

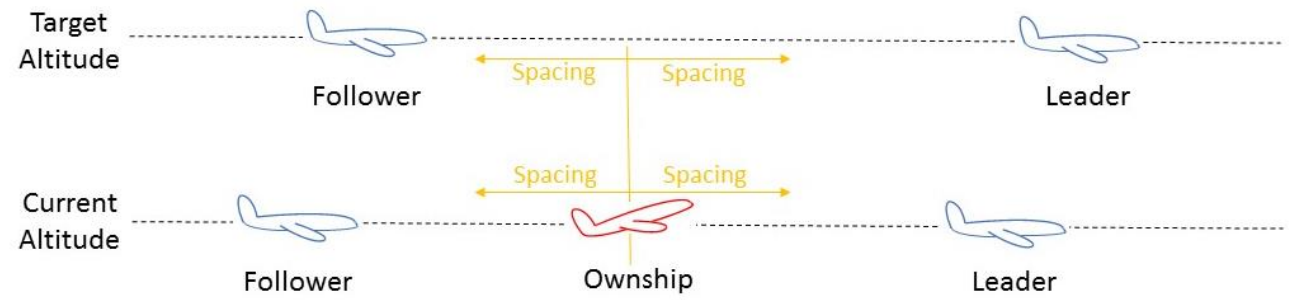

Figure 5. On-Airway Airborne Spacing with Altitude Change

The ownship aircraft would be responsible for maintaining at least the specified distances from all four target aircraft during the climb maneuver until the ownship is established at the target altitude. The controller would retain responsibility for separation from oncoming or crossing traffic between the original altitude and the target altitude. The ownship would be free to fly as fast or as slow as desired as long as the specified minimum distances from the four target aircraft are maintained, unless other constraints are put on the flight. Once the climb is completed, the pilot notifies the controller that the aircraft is established at the new altitude, and the controller terminates the aircraft's responsibility for spacing at the lower altitude.

\section{Discussion of Altitude-Change, On-Airway MMS Operation}

Airborne spacing at both the current and target altitudes during an altitude-change maneuver could significantly mitigate the impact on controller workload, allowing a busy controller to approve a climb that would otherwise be delayed because the controller does not have adequate time to monitor the climb spacing. However, the controller would be responsible for ensuring that oncoming or crossing traffic at the intermediate climb altitude would not conflict with the operation. Routine use of this procedure could have a significant impact on the ability of the controller to enable more fuel-efficient vertical trajectory performance without an unacceptable increase in workload. Traffic at different altitudes will likely be moving at slightly different speeds due to the wide variation in aircraft speeds for different aircraft types, plus the change in altitude means corresponding changes in winds, slight changes in Mach speed up to 40,000 ft, and there are likely slower aircraft flying at lower altitudes. If the pilot of the MMS-equipped aircraft uses onboard information, such as Cockpit Display of Traffic Information (CDTI) to wait for a gap so the climb will be feasible when it is request, this could also reduce the controller's workload in handling nuisance climb requests that must be delayed and then later granted.

The ability to routinely make timely step climbs to higher altitudes as fuel burns off has a significant benefit for airlines. The MMS algorithms could aid pilots in knowing when they could make requests for step climbs that are likely to be approved, since the pilot could check before making the request to see if there is space in the airway at the target altitude. In fact, this Situation Awareness information could aid the pilot in making timely requests for step climbs that are likely to be approved by the controller, even if an airborne MMS operation is not required for spacing. The use of airborne spacing during a climb could allow a busy controller to approve a climb into a tighter gap that would otherwise have been rejected, since the pilot would be responsible for monitoring spacing during the climb. If the aircraft has already been cleared for airborne spacing responsibility at the current altitude and has some leeway in 
making speed changes, in some cases the pilot could use the available information to position the aircraft under a gap at the target altitude so that a step climb could be approved when it is fuel optimal. A performance study would need to be conducted to see if the MMS-equipped aircraft positioning themselves for step climbs and climbing sooner have a negative impact on the ability for unequipped aircraft to make timely step climbs.

Previous research and development under PTM, In-Trail Procedure, and terminal area merging and spacing studied airborne spacing algorithms for altitude-change operations in depth and learned much that is relevant to this operation, including algorithm development, clearance messaging, and safety analysis. Previous research has shown that airborne spacing during altitude-change operations must include a buffer to account for speed variations that occur during climbing and descending, which vary significantly with aircraft type, FMS functionality, pilot procedure, and aircraft weight. These speed variations happen quickly and are difficult to predict or capture in messaging. Thus, the procedure would likely be limited to a single aircraft performing an airborne altitude-change procedure between target Leader and Follower aircraft that are flying level at the initial and target altitudes, at least in the near- to mid-term TBO environment.

The spacing algorithm must be capable of determining whether the maneuver is feasible, assuming the four target aircraft will maintain their current speeds, with a small added buffer for speed variability. Due to ADS-B range issues, the onboard system may not be capable of determining whether there is oncoming or crossing traffic at an intermediate altitude that would preclude the operation, so this must be checked by the controller before issuing the clearance.

\section{Off-Airways MMS Operations for Crossing and Merging onto Airways}

This section examines MMS operations that could reduce controller workload for managing aircraft that are operating off of the established airways for a short or long period of time, including crossing an airway or merging laterally or vertically onto an airway.

\section{Single-Altitude, Airborne Merging and Spacing onto an Airway}

A controller managing an aircraft that has been operating off of the established airways system could clear the aircraft for airborne merging and spacing onto an airway. The waypoint at which the merge is to take place would be designated in the clearance. The ownship aircraft may be cleared for airborne spacing behind a Leader, in front of a Follower, or both, as shown in Figure 6. Since the ownship aircraft is not on the same heading as the target aircraft, the spacing is based on relative timing to the merge point. The operation could be initiated before the ownship has achieved the designated spacing range (for example when the relative distance to the merge point between ownship and Leader is smaller than the designated spacing) as long as the two aircraft are sufficiently laterally separated and the ownship is projected to achieve the correct spacing before lateral separation is lost between his course and the airway. Acceptance of the airborne spacing clearance would connote the pilot affirming his ability to obtain the required spacing (based on guidance from onboard algorithms). Once established on the airway, the controller could then issue a clearance for the ownship to maintain airborne spacing along the airway, or the merging clearance could automatically transition to airborne spacing on the airway.

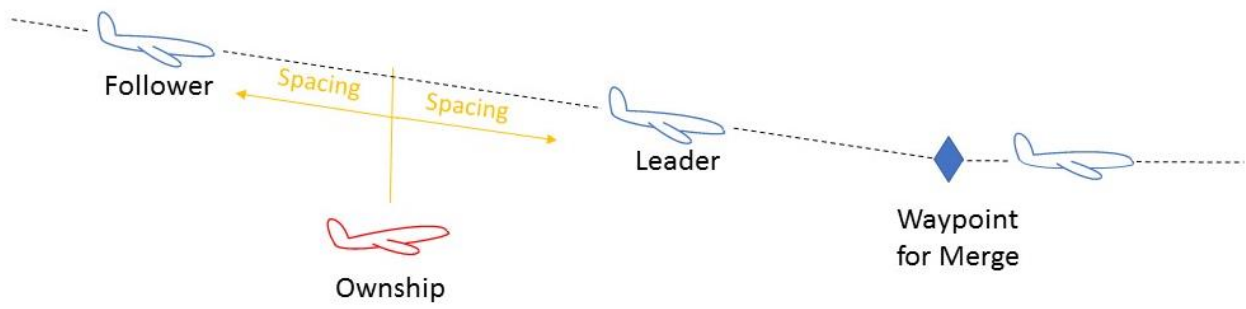

Figure 6. Airborne Merging and Spacing onto Airway

\section{Single-Altitude, Spacing Operation for Crossing an Airway}

An aircraft that is operating off of the established airways system could be cleared for airborne spacing for crossing an established airway by maintaining designated spacing between a Leader aircraft and Follower aircraft that are on an established airway, as shown in Figure 7. The spacing is based on relative time to the point at which the ownship will cross the airway, and the ownship is responsible for both lateral separation and longitudinal spacing from both target aircraft. The controller would have to establish and maintain sufficient spacing between the Leader and Follower aircraft to accommodate the crossing ownship. 
The operation could begin before the ownship has achieved the designated spacing range (for example when the relative distance to the crossing point between ownship and Leader is smaller than the designated spacing) as long as the two target aircraft are sufficiently laterally separated and the ownship is projected to achieve the correct spacing before lateral separation is lost between the converging airways. As always, the ownship pilot would have to accept the airborne spacing clearance, which affirms his ability to obtain the required spacing (based on guidance from onboard algorithms).

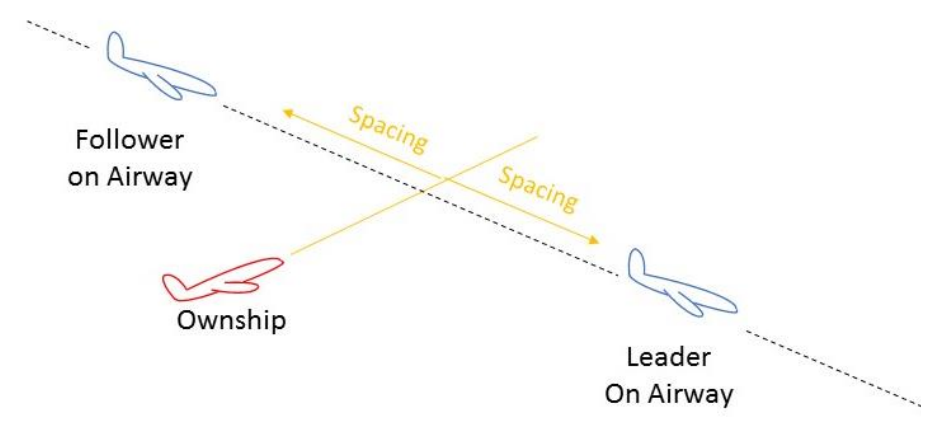

Figure 7. Airborne Spacing for Crossing an Airway

\section{On-Airway Airborne Spacing with Altitude-Change Operation}

This operation could be used to merge a climbing aircraft into a dense en route stream on an airway, with the aircraft performing airborne spacing at the target altitude between a Leader and a Follower target aircraft, as shown in Figure 8. The relative spacing is based on the longitudinal distance between the two aircraft. The controller is responsible for ensuring that a gap is maintained at the target altitude.

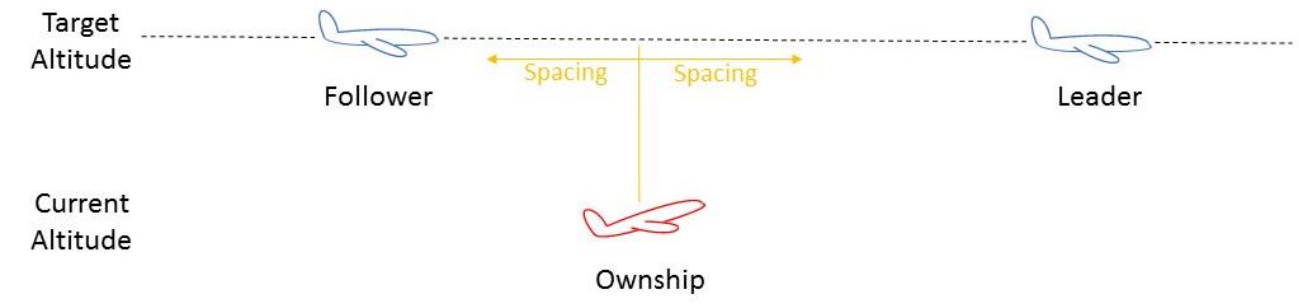

Figure 8. On-Airway Airborne Spacing with Altitude Change

The ownship aircraft would be responsible for maintaining at least the specified distances from the two target aircraft during the climb maneuver until the ownship is established at the target altitude. Once the climb is completed, the pilot notifies the controller that the aircraft is established at the new altitude, and the controller terminates the operation, issuing a separate clearance to maintain spacing on the airway if desired. The controller retains responsibility for separation from all other aircraft, including oncoming aircraft at intermediate altitudes during the operation.

\section{Discussion of MMS Operations for Crossing and Merging onto Airways}

The operations in this section expand merging and spacing functionality to aircraft that are operating off of airways. The altitude-change operation for merging onto an airway could be used routinely to reduce controller workload for aircraft that are merging into the en route stream after takeoff and climb out. When an aircraft in low- to mediumdensity airspace requests a clearance to fly direct to a near or far waypoint, the approval of the route is at the controller's discretion and is based primarily on current and predicted controller workload and operational norms. Clearing an off-airways aircraft to use airborne spacing to cross established airways and to merge back onto the airway at a designated merge point could make it much more likely that a controller would approve the operation. Some of the operational scenarios for which off-airways merging and spacing operations could also be used to reduce controller workload include:

- merging aircraft into the en route stream after takeoff and climb out,

- managing aircraft that have deviated from the airway to avoid hazardous weather, and

- managing aircraft that have been approved for a lateral offset maneuver to pass a slower aircraft. 
Implementation and regular use of airborne off-airway merging and spacing operations may yield enough operational benefits in optimized routing to airlines to justify equipage decisions. Previous research during development of the PTM concept have examined the performance and safety aspects of performing airborne spacing on merging and intersecting tracks for a limited range of angles and including initial studies relevant to lateral offset maneuvers. Much research and development would need to be completed to implement these operations on highcapacity airways in en route airspace, but many of the basic feasibility questions have already been answered.

\section{Strategic Metering Operations}

Strategic metering operations can be used to manage the flow of aircraft into capacity-constrained regions throughout the NAS. A single flight might be subject to metering into a high-density en route stream after takeoff, followed by metering into one or more en route FCAs due to convective weather, and finally metering into the destination terminal area. Each of these instances of TOAC may involve a different size of time window, reflecting the precision required for flow management at that point. Ground-based metering will be used for aircraft that are not equipped to perform flight-deck-based metering. Pilots could perform metering using their FMS RTA capability or using guidance from an algorithm implemented on an EFB. Current FMSs can only manage to a single RTA; however, once each RTA point is reached, the pilot can enter the next RTA to be met as long as the RTAs are suitably separated in time and are compatible in timing such that meeting an earlier RTA does not make a later one infeasible. RTAs could also be reassigned dynamically during a flight if unexpected delays arise or shortcuts become available. If the timing to meet multiple TOAC points requires excessive alternating between speeding up and slowing down, this will not be accepted by the airlines in routine operations.

Much research is required to identify how TOAC can be applied to effectively manage flows in the NAS. One key question is how far ahead a TOAC window should be set. Should TOAC windows be assigned preflight, which may be five or more hours ahead and then adjusted as needed to match evolving capacity? The recent operational deployment of TBFM in the NAS has shown that using merging and spacing to condition the flow of aircraft approaching the extended terminal area is extremely complex to implement, involving the use of multiple freeze horizons to control the dense flows of traffic while accommodating short-haul flights taking off within the outer freeze horizons and merging into the streams.

The use of TOAC to manage the flow of traffic headed towards a capacity-constrained destination has the potential to dramatically change the flow of traffic. Theoretically, in far-term TBO, a pilot issued a TOAC window for the destination could choose how early to take off and how economically to fly to arrive at the designated merge point within the TOAC window, knowing that his arrival would be smoothly and efficiently accommodated into the arrival stream. However, this involves a high level of trust between the operators and the service providers, and the establishment of policies to deal with contingencies, both inadvertent and willful. The operator must be trusted to arrive within the TOAC window and not show up too early, causing a headache for the controller. If exceptionally early aircraft are accepted for arrivals ahead of their TOAC window, then operators will be rewarded for improper behavior.

Additionally, the system must be stable and predictable enough that unexpected en route delays seldom arise to jeopardize an aircraft's TOAC performance. Once an aircraft is airborne, an FMS can reliably predict the time an aircraft will reach an RTA five hours away within a few minutes, if there are no disruptions or deviations to the flight. However, if the aircraft is subject to multiple TOACs throughout the flight and is conducting the tactical merging and spacing operations discussed in previous sections, the timing of delivery to the destination TOAC is far from certain. The following section discusses interdependencies between multiple MMS operations.

\section{Interdependencies Between Multiple MMS Operations}

As discussed in previous sections, an individual flight may be simultaneously subject to both a tactical MMS operation to aid the controller in managing the local flow, and one or more strategic MMS operations. This section addresses interdependencies between multiple MMS operations and opportunities for making up time after encountering delays during earlier phases of flight. Referring back to the example flight profile described in Section II, the five-hour flight includes two step climbs separated by slightly more than an hour. It is likely that the flight will encounter a variety of traffic densities as the flight progresses. In low-density airspace, there should be ample opportunities to fly at a desired speed. In medium density airspace, any delays encountered will hopefully be limited to short duration, especially since the flight will only be operating at a given altitude for about one hour. Using the assumption that the acceptable speed range is centered around the planned, fuel-efficient speed for the flight, any delays encountered such as spacing behind a slower aircraft in one segment of the flight, could likely be recovered by flying at a faster speed for another segment of the flight, such as after the next step climb or after turning onto a 
different airway. For the portions of the flight that are traversing high-density airspace, the operator is likely to have few options to make up for or absorb delays, and this lack of flexibility should be taken into account when planning TOAC windows for flights that are predominately operating in high-density flows. In general, since some amount of delay is expected during a flight through high-density airspace, it may be prudent for an aircraft to operate during the early phases of the flight aiming to arrive at or even slightly ahead of the early limit of the assigned TOAC so that after encountering the expected delays the flight will still be able to arrive within the TOAC. The TOAC windows may also be allocated slightly above the predicted capacity to apply some pressure to the capacity-constrained resource so that if the actual capacity is larger than the forecast, capacity is not wasted.

If the controller were aware of the TOAC window for each flight and how much the aircraft is behind or ahead of the TOAC window, then as the controller absorbs delays and resolves conflicts, he could show minor preferences for aircraft that are behind schedule, such as using a path stretch for an aircraft that is ahead of schedule while allowing an aircraft that is behind schedule to pass them. This would be a difficult task for busy controllers to manage in highdensity airspace, but could be feasible if the controller was aided by decision-support automation designed to handle this function. If controllers in medium- and low-density airspace were aware of TOAC requirements for a flight, they could likely find many opportunities to expedite the flow for flights that need to make up for prior delays.

Aircraft traveling in medium-density airspace along predefined airways have some flexibility to increase speed to make up for delays. If behind an aircraft flying at a slower speed, the pilot can use CDTI and onboard spacing tools to look for opportunities to request an altitude change that may allow operation at increased speed. As other aircraft depart the airway to head to other destinations, there may be opportunities to increase speeds. NASA has prototyped an onboard tool, Traffic Aware Strategic Aircrew Requests (TASAR) ${ }^{29}$, that can aid the pilot in identifying minor lateral and/or vertical route changes to decrease flight time and/or save fuel, which can be requested via voice or datalink. TASAR will only recommend route changes that are clear of hazardous weather, avoid active Special Use Airspace, and are free of near-term traffic conflicts so that they are likely to be accepted by controllers. A procedure that could be used more frequently under TBO to allow passing along an airway is the use of a lateral offset maneuver $^{30}$. In this maneuver, an aircraft is cleared by a controller to deviate from the airway and fly laterally at a specified number of miles to the left or right of the airway, rejoining the airway at a future point. Controller workload to monitor this maneuver could be reduced if the pilot were to perform an airborne merging and spacing operation when rejoining the airway.

In today's environment, an aircraft arriving at a major airport is typically routed to the end of a dense queue of traffic heading towards the destination. The implementation of TBO operations, such as MMS, may result in shortening these dense queues of traffic and allowing more geographically disperse merging towards major airports, but these queues are unlikely to disappear in the foreseeable future. Once an aircraft enters the dense queue, there are few opportunities for passing other aircraft and making up for prior delays, so the assignment of TOAC windows should probably be at or near the projected start of the queue.

For en route FCAs, such as due to hazardous weather blocking multiple airways, it can be assumed that the airspace leading up to the region would be able to handle traffic at or near the nominal sector capacity and would not be overloaded by the traffic moving through it towards the FCA. In this case, maneuvering to meet a TOAC window may be feasible right up to the boundary of the FCA, and the TOAC point would be near the FCA boundary. The TOAC should naturally condition the rate of flow of streams of traffic approaching the FCA.

\section{Concluding Remarks}

Airborne- and ground-based MMS operations will play a major role in the transition to TBO. The use of MMS operations in a TBO environment for strategic traffic flow management as well as tactically to ensure smooth flows and to increase capacity and flexibility was examined. An example set of likely tactical airborne MMS operations were identified that could be used by aircraft that are on established airways or by aircraft utilizing optimized, aircraftspecific routing. The on-airways operations may reduce controller workload in monitoring aircraft on airways, possibly enabling the introduction of additional airways to increase sector capacity. The off-airways operations are designed to increase opportunities for optimized, aircraft-specific routing by reducing the controller workload impact of monitoring off-airways aircraft that are crossing airway traffic and merging back onto airways. Interdependencies between multiple tactical and strategic MMS operations, such as timing issues, were also addressed. This study, which leverages previous research findings, is an initial step toward defining a candidate set of functional requirements for onboard and ground-based tools and procedures to enable a suite of MMS operations appropriate for different situations and operational goals across the en route, departure, and arrival phases of flight. 


\section{Acknowledgments}

This work was conducted under NASA LITES II contract NNL15AA03B. I am grateful to Bryan Barmore and the researchers on the Trajectory-Based Operations team at NASA Langley Research Center who have participated in shaping the TBO concept and provided feedback on the MMS operations described in this paper.

\section{References}

${ }^{1}$ Johnson, Sally C., Barmore, Bryan E., "NextGen Far-Term Concept Exploration for Integrated Gate-to-Gate Trajectory-Based Operations," 16 ${ }^{\text {th }}$ AIAA Aviation Technology, Integration, and Operations Conference, Washington, D.C., 13-17 June, 2016.

${ }^{2}$ NextGen Joint Planning and Development Office, "Concept of Operations for the Next Generation Air Transportation System," Version 3.2, 2011.

${ }^{3}$ NextGen Joint Planning and Development Office, "JPDO Trajectory-Based Operations (TBO) Study Team Report,” 2011.

${ }^{4}$ Single European Sky ATM Research Consortium, “SESAR Concept of Operations,” DLT-0612-222-01-00, 2007.

${ }^{5}$ Evans, Antony D., and Lee, Paul, “Analyzing Double Delays at Newark Liberty International Airport,” 16 ${ }^{\text {th }}$ AIAA Aviation Technology, Integration, and Operations Conference, Washington, D.C., 13-17 June, 2016.

${ }^{6}$ Wichman, K. D., Carlsson, G., and Lindberg, G. V., "Flight Trials 'Runway-to-Runway' Required Time of Arrival Evaluations for Time-Based ATM Environment," 20th Digital Avionics Systems Conference, Daytona Beach, FL, 2001.

${ }^{7}$ Wichman, K. D., J. K., Bleeker, O. F., and Rademaker, R. M., "Flight Validations of Downlinked Flight Management System 4D Trajectory," 26th Digital Avionics Systems Conference, Dallas, TX, 2007.

${ }^{8}$ Klooster, J. K., Wichman, K. D., and Bleeker, "4D Trajectory and Time of Arrival Control to Enable Continuous Descent Arrivals," AIAA Guidance, Navigation, and Control Conference, Honolulu, HI, 2008.

${ }^{9}$ Klooster, J., DelAmo, A., and Manzi, P., "Controlled Time of Arrival Flight Trials - Results and Analysis," Eighth USA/Europe Air Traffic Management Research and Development Seminar, Napa, CA, 2009.

${ }^{10}$ Wynnyk, C., Balakrishna, M., MacWilliams, P., and Becher, T., "2011 Trajectory Based Operations Flight Trials," Tenth USA/Europe Air Traffic Management Research and Development Seminar, Chicago, IL, 2013.

${ }^{11}$ Mutuel, L.H., Neri, P., and Paricaud, E., "Initial 4D Trajectory Management Concept Evaluation," Tenth USA/Europe Air Traffic Management Research and Development Seminar, Chicago, IL, 2013.

${ }^{12}$ Barmore, B., Abbott, T., \& Krishnamurthy, K., "Airborne-managed Spacing in Multiple Arrival Streams," 24th International Congress of the Aeronautical Sciences (ICAS 2004), Yokohama, Japan, 2004.

${ }^{13}$ Barmore, B., "Airborne Precision Spacing: A Trajectory-Based Approach to Improve Terminal Area Operations," 25th IEEE/AIAA Avionics Systems Conference, 2006.

${ }^{14}$ Barmore, B. E., Bone, R. S., and Penhallegon, W. J, "Flight deck-based merging and spacing operations," Air Traffic Control Quarterly Vol. 17, No. 1, 2009.

${ }^{15}$ Hicok, Daniel S., and Barmore, Bryan E., "Concept of Operations for Interval Management Arrivals and Approach," AIAA Guidance, Navigation, and Control Conference, 2016.

${ }^{16}$ RTCA. "Safety, Performance and Interoperability Requirements Document for Airborne Spacing - Flight Deck Interval Management (ASPA-FIM) (DO-328A)" RTCA, Washington, DC, 2015.

${ }^{17}$ Chittargi, K. Martin, L., "Time Based Flow Management (TBFM) as a Service-With NextGen SOA," Integrated Communications, Navigation and Surveillance Conference, Washington, DC, April 2013.

${ }^{18}$ Swenson, H. N., Hoang, T., Engelland, S., Vincent, D., Sanders, T., Sanford, B., Heere, K., "Design and Operational Evaluation of the Traffic Management Advisor at the Fort Worth Air Route Traffic Control Center," Proceedings of the 1st USA/Europe Air Traffic Management Research and Development Seminar, Saclay, France, June 1997.

${ }^{19}$ Baxley, B., Swenson, H., Prevot, T., Callantine, T., "NASA's ATM Technology Demonstration-1: Integrated Concept of Arrival Operations," 31st Digital Avionics Systems Conference, Williamsburg, VA, October 2012.

${ }^{20}$ Baxley, Brian T., Johnson, William C., Scardina, John, and Shay, Richard F.. "Air Traffic Management Technology Demonstration-1 Concept of Operations (ATD-1 ConOps), Version 3.0." NASA TM-2016-219213, 2016.

${ }^{21}$ U.S. Department of Transportation Office of Inspector General, "FAA Has Not Effectively Deployed Controller Automation Tools That Optimize Benefits of Performance-Based Navigation,” Audit Report AV-2015-081, August, 2015.

${ }^{22}$ Murdoch, J. L., Bussink, F. J., Chamberlain, J. P., Chartrand, R. C., Palmer, M. T., and Palmer, S. O., "Enhanced Oceanic Operations Human-In-The-Loop In-Trail Procedure Validation Simulation Study,” NASA TP-2008-215313, 2008.

${ }^{23}$ Bussink, F. J., Murdoch, J. L., Chamberlain, J. P., and Jones, K. M, "Pilot In-Trail Procedure Validation Simulation Study," 8th AIAA Aviation Technology, Integration, and Operations Conference, Anchorage, Alaska, 2008.

${ }^{24}$ Chartrand, R. C., Bussink, F. J., Graff, T. J., Murdoch, J. L., and Jones, K. M., “Operational Improvements From the In-Trail Procedure in the North Atlantic Organized Track System," 8th AIAA Aviation Technology, Integration, and Operations Conference, Anchorage, Alaska, 2008.

${ }^{25}$ Chartrand, Ryan C., Hewitt, Katrin P., Sweeney, Peter B., Graff, Thomas J., and Jones, Kenneth M., "In-Trail Procedure Air Traffic Control Procedures Validation Simulation Study,” NASA TM-2012-217327, 2012.

${ }^{26}$ Jones, K. M., Graff, T. J, Chartrand, R. C., Carreño, V. C., and Kibler, J. L. (in preparation). Pairwise Trajectory Management (PTM): Concept Overview, NASA TM. 
${ }^{27}$ Jones, K. M., "Pair-Wise Trajectory Management-Oceanic (PTM-O): Concept of Operations-Version 3.9," NASA TP-2014$218188,2014$.

${ }^{28}$ Jones, K.M., Graff, T.J., Chartrand, R.C., Carreño, V.C., and Kibler, J.L., "Pairwise Trajectory Management (PTM): Concept Overview," 17 $7^{\text {th }}$ AIAA Aviation Technology, Integration, and Operations Conference, Denver, CO, June 5-9, 2017.

${ }^{29}$ Ballin, M. G., Wing, D. J., "Traffic Aware Strategic Aircrew Requests (TASAR)," 12th AIAA Aviation Technology, Integration, and Operations Conference, Indianapolis, IN, September 2012.

${ }^{30}$ Lauderdale, T. A., Santiago, C., and Pankok, C., "A Computer Simulation of the System-Wide Effects of Parallel-Offset Route Maneuvers," AIAA Guidance, Navigation, and Control Conference and Modeling and Simulation Technologies

Conference, Toronto, Canada, August 2-5, 2010. 